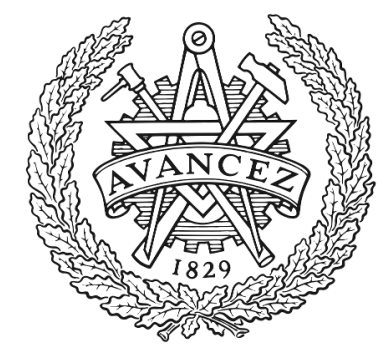

CHALMERS

UNIVERSITY OF TECHNOLOGY

\title{
Semi-Passive 3D Positioning of Multiple RIS-Enabled Users
}

Downloaded from: https://research.chalmers.se, 2023-04-26 10:17 UTC

Citation for the original published paper (version of record):

Keykhosravi, K., Keskin, F., Dwivedi, S. et al (2021). Semi-Passive 3D Positioning of Multiple RIS-Enabled Users. IEEE Transactions on Vehicular Technology, 70(10): 11073-11077. http://dx.doi.org/10.1109/TVT.2021.3109786

N.B. When citing this work, cite the original published paper.

(O2021 IEEE. Personal use of this material is permitted.

However, permission to reprint/republish this material for advertising or promotional purposes 


\title{
RIS-Enabled Semi-Passive Multi-User 3D Localization and Doppler Estimation
}

\author{
Kamran Keykhosravi, Member, IEEE, Musa Furkan Keskin, Member, IEEE, Satyam Dwivedi, Member, IEEE, \\ Gonzalo Seco-Granados, Senior Member, IEEE, and Henk Wymeersch, Member, IEEE.
}

\begin{abstract}
Reconfigurable intelligent surface (RIS), operating as a lens or a reflector, is set to be a revolutionary technology in the 6th generation of wireless systems. With most works that consider RISs as reflectors, the RIS provides a non lineof-sight (NLOS) path between the base station and the user. In this letter, we study the application of RIS in a multi-user passive localization scenario, where RIS is mounted on the user side, providing NOLS paths between a transmitter and multiple asynchronous receivers. We show that user's 3D position can be estimated with submeter accuracy in a large area around the transmitter, using LOS and NLOS time-of-arrival measurements at the receivers. We do so, by developing the signal model, deriving the Cramér-Rao bounds, and devising an estimator that attains the bounds. Furthermore, by properly adjusting the RIS phase profiles, we circumvent inter-path interference.
\end{abstract}

Index Terms-Reconfigurable intelligent surfaces, passive localization, Cramér-Rao lower bounds

\section{INTRODUCTION}

Realization of smart radio environments empowered by reconfigurable intelligent surfaces (RISs), which enables wideranging communication and radio sensing with high energy and spectrum efficiency, is one of the foremost ambitions of the sixth generation of wireless systems [1]. RIS consists of a multitude of unit cells, whose response to the impinging electromagnetic wave can be controlled, and thereby can improve the quality and coverage of wireless communication and also enable or improve radio localization [2], [3]. In addition to these benefits, RISs are semi-passive devices with low cost, which make them ideal to be mounted on vehicles.

Radio localization has attracted increasing attention in recent years as technologies such as millimeter wave, multipleinput multiple-output (MIMO), and RIS enable high-accuracy positioning of users, based on the time-of-arrival (ToA) and angles of arrival and departure measurements. Considering the nature of the user, localization techniques can be categorized into active and passive methods. While with the former case the user transmits or receives signals, in the latter one, the user only reflects the signals from transmitter (Tx). Many studies

This work was supported, in part, by the Swedish Research Council under grant 2018-03701, the Marie Skłodowska-Curie Individual Fellowships (H2020-MSCA-IF-2019) Grant 888913 (OTFS-RADCOM), the Spanish Ministry of Science, Innovation and Universities under Projects TEC2017-89925$\mathrm{R}$ and PRX18/00638 and by the ICREA Academia Programme.

K. Keykhosravi, M. Keskin, and H. Wymeersch are with the Department of Electrical Engineering at Chalmers University of Technology, 41296 Gothenburg, Sweden (e-mail: kamrank@chalmers.se).

G. S. Granados is with the Department of Telecommunications and Systems Engineering, Universitat Autònoma de Barcelona, 08193 Barcelona, Spain.

S. Dwivedi is with Ericsson Research, Linkoping, Sweden. have been conducted on passive localization based on a variety of approaches such as radio-frequency identification (RFID) [4], [5], signal eigenvector [6], received signal strength (RSS) [7], and ToA-based passive positioning. With the latter case, which is the focus of this letter, the user location is estimated based on the received signal ToA at multiple receivers (Rxs). This topic has been studied in two-dimensional space under the assumption of synchronous [8], quasi-synchronous [9], and asynchronous networks [10]. In [11], the authors study the 2D localization performance of a joint radar and RFID system. Moreover, bistatic ToA estimation has been investigated in passive sensing systems that employ the signals transmitted by illuminators of opportunity (IO) [12]. To the best our knowledge, this is the first paper on RIS-enabled passive localization.

In this letter, we investigate the multi-user 3D positioning problem, employing a single-antenna Tx and multiple singleantenna Rxs, where each user is equipped with a RIS (see Fig. 1). We propose a low-complexity positioning algorithm, which utilizes orthogonal sequences in the design of RIS phase profiles. By employing the orthogonality property of the received signal, the algorithm can resolve multipath interference and data association problem. In other words it can decompose the received signal at each $\mathrm{Rx}$ to the line-of-sight (LOS) component and signals reflected from each user equipment (UE). Thereafter, the ToA can be readily estimated at each $\mathrm{Rx}$ for each of the multipath components, which enables localization of the UEs. Finally, we evaluate the localization error for the proposed method and show that it reaches the theoretical Cramér-Rao lower bounds (CRB).

\section{A. Notation}

Vectors, which are columns, are shown by bold lower-case letters and matrices by bold upper-case ones. The element at the $i$ th row and the $j$ th column of the matrix $\boldsymbol{A}$ is shown as $[\boldsymbol{A}]_{i, j}$. The set $\mathbb{C}$ and $\mathbb{T}$ represents the set of complex numbers and all the complex numbers with unit magnitude, respectively.

\section{System Model}

\section{A. Signal Model}

We consider one Tx (a base station (BS)) with location $\boldsymbol{p}_{0}$ and $M$ Rxs with locations $\boldsymbol{p}_{1}, \ldots, \boldsymbol{p}_{M}$, as well as $N$ UEs with locations $\boldsymbol{x}_{1}, \ldots, \boldsymbol{x}_{N}$. Each of the UEs is equipped with a RIS, while the Tx and Rxs have a single antenna. The Rxs are not synchronized with the BS and have clock biases 


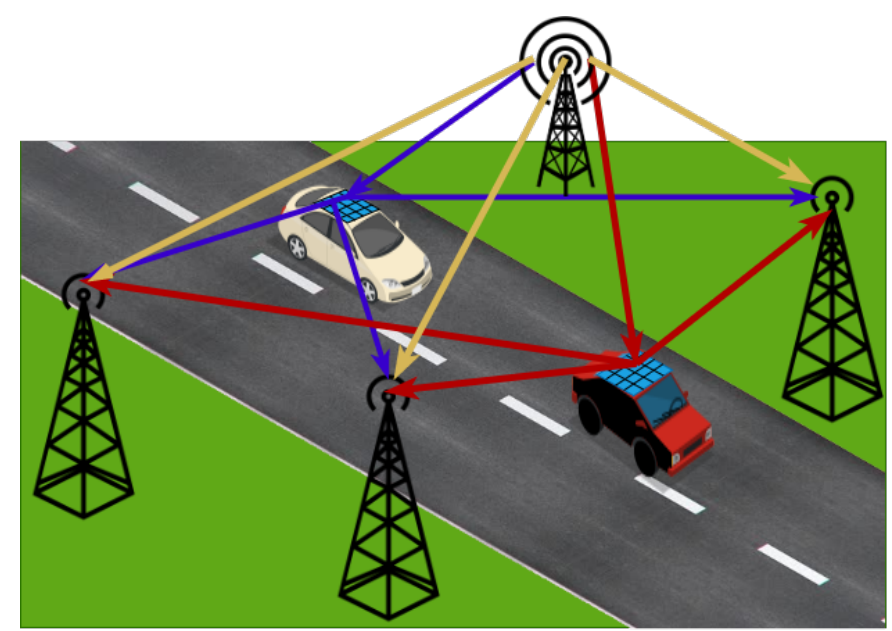

Fig. 1. A schematic of the system model for two UEs $(N=2)$ and three $\operatorname{Rxs}(M=3)$.

$B_{1}, \ldots, B_{M}$. We assume transmission of $T$ OFDM symbols with $K$ subcarriers during each localization occasion.

The signal received at the $m$ th $\mathrm{Rx}$ can be represented by the matrix $\boldsymbol{Y}_{m} \in \mathbb{C}^{K \times T}$. Assuming constant transmission over all subcarriers, we have

$$
\boldsymbol{Y}_{m}=\sqrt{E_{\mathrm{s}}} \sum_{n=0}^{N} \boldsymbol{d}\left(\tau_{n, m}\right) \boldsymbol{\alpha}_{n, m}^{\top}+\boldsymbol{W}_{m},
$$

where $E_{\mathrm{S}}$ is the symbol's energy and

$$
\boldsymbol{d}(\tau)=\left[1, e^{\jmath 2 \pi \Delta f \tau}, \ldots, e^{\jmath 2 \pi(K-1) \Delta f \tau}\right]^{\top}
$$

is a steering vector across subcarriers, where $\Delta f$ is the subcarrier spacing. For the LOS path $(n=0)$, the delay is $\tau_{0, m}=\left\|\boldsymbol{p}_{0}-\boldsymbol{p}_{m}\right\| / c+B_{m} / c$, in which the distance $\left\|\boldsymbol{p}_{0}-\boldsymbol{p}_{m}\right\|$ is known and $c$ is the speed of light. For the reflected paths $(n>0)$, the delay is

$$
\tau_{n, m}=\frac{\left\|\boldsymbol{p}_{0}-\boldsymbol{x}_{n}\right\|+\left\|\boldsymbol{x}_{n}-\boldsymbol{p}_{m}\right\|+B_{m}}{c} .
$$

The vector $\boldsymbol{\alpha}_{n, m} \in \mathbb{C}^{T \times 1}$ represents the complex gain of different paths. For $n=0$ (LOS) $\boldsymbol{\alpha}_{0, m}=\alpha_{0, m} \mathbf{1}_{T}$, where $\alpha_{0, m}$ indicates the LOS gain. For $n \neq 0$, we have

$$
\left[\boldsymbol{\alpha}_{n, m}\right]_{t}=\gamma_{n, 0} \gamma_{n, m} \boldsymbol{a}\left(\boldsymbol{\theta}_{n, m}\right)^{\mathrm{H}} \boldsymbol{\Omega}_{n}[t] \boldsymbol{a}\left(\boldsymbol{\phi}_{n, 0}\right),
$$

in which $\gamma_{n, 0}$ is the complex channel gain from the transmitter to UE $n$ and $\gamma_{n, m}$ is the complex channel gain from UE $n$ to receiver $m$. The noise matrix is represented by $\boldsymbol{W}_{m} \in$ $\mathbb{C}^{K \times T}$, which has iid circularly-symmetric Gaussian elements and variance $N_{0}$.

Moreover, $\boldsymbol{a}\left(\boldsymbol{\theta}_{n, m}\right)$ is the steering vector of the RIS of $n$th UE at angle-of-departure (AoD) $\boldsymbol{\theta}_{n, m}$, measured in the unknown frame of reference of UE $n$. Let $\boldsymbol{R}_{n}$ indicate the unknown rotation matrix mapping the global frame of reference to the coordinate system associated with the $n$th RIS. Then AoD $\boldsymbol{\theta}_{n, m}$ represents the $3 \mathrm{D}$ angle in the direction of vector $\boldsymbol{w}_{n, m}=\boldsymbol{R}_{n}\left(\boldsymbol{p}_{m}-\boldsymbol{x}_{n}\right)$, i.e., $\boldsymbol{\theta}_{\mathrm{az}}=\operatorname{atan} 2\left(\left[\boldsymbol{w}_{n, m}\right]_{2},\left[\boldsymbol{w}_{n, m}\right]_{1}\right)$ and $\boldsymbol{\theta}_{\mathrm{el}}=\operatorname{acos}\left(\left[\boldsymbol{w}_{n, m}\right]_{3} /\left\|\boldsymbol{w}_{n, m}\right\|\right.$. Similarly, $\boldsymbol{\phi}_{0, n}$ indicates the $n$th RIS steering vector at angle-of-arrival (AoA) $\phi_{0, n}$, which is the 3D angle associated with the vector $\boldsymbol{v}_{n, 0}=$ $\boldsymbol{R}_{n}\left(\boldsymbol{x}_{n}-\boldsymbol{p}_{0}\right)$. The steering vector at angle $\boldsymbol{\psi}$ for an $W_{\mathrm{r}} \times W_{\mathrm{c}}$ RIS with distance $d$ between adjacent elements is $\boldsymbol{a}(\boldsymbol{\psi})=$ $\boldsymbol{a}_{\mathrm{r}}(\boldsymbol{\psi}) \otimes \boldsymbol{a}_{\mathrm{c}}(\boldsymbol{\psi})$, where

$$
\begin{aligned}
& \boldsymbol{a}_{\mathrm{r}}(\boldsymbol{\psi})=e^{\jmath \beta_{\mathrm{r}}}\left[1, e^{\jmath d[\boldsymbol{k}(\boldsymbol{\psi})]_{1}}, \ldots, e^{\jmath\left(W_{\mathrm{r}}\right) d[\boldsymbol{k}(\boldsymbol{\psi})]_{1}}\right]^{\top} \\
& \boldsymbol{a}_{\mathrm{c}}(\boldsymbol{\psi})=e^{\jmath \beta_{\mathrm{r}}}\left[1, e^{\jmath d[\boldsymbol{k}(\boldsymbol{\psi})]_{2}}, \ldots, e^{\jmath\left(W_{\mathrm{r}}\right) d[\boldsymbol{k}(\boldsymbol{\psi})]_{2}}\right]^{\top}
\end{aligned}
$$

where $\beta_{\mathrm{r}}=-\left(W_{\mathrm{r}}-1\right) d[\boldsymbol{k}(\boldsymbol{\psi})]_{1} / 2$ and $\beta_{\mathrm{c}}=-\left(W_{\mathrm{c}}-\right.$ 1) $d[\boldsymbol{k}(\boldsymbol{\psi})]_{2} / 2$ and

$$
\boldsymbol{k}(\boldsymbol{\psi})=\frac{2 \pi}{\lambda}\left[\sin \psi_{\mathrm{el}} \cos \psi_{\mathrm{az}}, \sin \psi_{\mathrm{el}} \sin \psi_{\mathrm{az}}, \cos \psi_{\mathrm{el}}\right]^{\top}
$$

is the wavenumber vector. Finally, $\boldsymbol{\Omega}_{m}[t] \in \mathbb{T}^{W \times W}$, where $W=W_{\mathrm{r}} W_{\mathrm{c}}$, is a diagonal matrix that represents the phase profile of RIS $n$ as a function of time $t$.

\section{B. Problem Formulation}

Our goal in this letter is to estimate the locations of the $N$ UEs, $\boldsymbol{x}_{1}, \ldots, \boldsymbol{x}_{N}$. To do this, we formulate the following objectives:

- To estimate at $\operatorname{Rx} m$, the $N$ ToAs $\tau_{n, m}$. For this, we use the design freedom of the RIS in terms of $\boldsymbol{\Omega}_{n}[t]$ to avoid interference from different paths.

- To compute time-difference-of-arrival (TDoA) measurements at each of the $M \mathrm{Rxs}$ and process them jointly to localize all users.

\section{MEthodology}

In this section, we address the two steps mentioned in Section II-B We first introduce a special RIS phase profile design in Section III-A that allows us to decouple the received signals at each Rx. Then based on the received signals we estimate ToAs in Section III-B Finally, in Section III-C we use the ToAs to estimate the position of the UEs.

\section{A. RIS phase profile design}

In this section, we design the phase profile of each RIS to avoid the interference between different signal paths. To do so, for UE $n$, we set the RIS profile $\Omega_{n}[t]$ to be a multiplication of a static diagonal matrix $\boldsymbol{\Omega}_{n} \in \mathbb{T}^{W \times W}$ and a dynamic scalar $\left[\boldsymbol{\omega}_{n}\right]_{t} \in \mathbb{T}$, i.e., $\boldsymbol{\Omega}_{n}[t]=\left[\boldsymbol{\omega}_{n}\right]_{t} \boldsymbol{\Omega}_{n}$. We also define $\boldsymbol{\omega}_{0}=\mathbf{1}_{T}$. As shown in Section III-B, we can avoid inter-path interference if the vectors $\boldsymbol{\omega}_{n} \in \mathbb{T}^{T \times 1}$ for $n=0,1, \ldots, N$ form an orthonormal set, i.e.,

$$
\boldsymbol{\omega}_{n}^{H} \boldsymbol{\omega}_{n^{\prime}}= \begin{cases}T & \text { if } n=n^{\prime} \\ 0 & \text { otherwise }\end{cases}
$$

Therefore, one should set the the number of transmission $T$ higher than $N$, to be able to select $N+1$ orthonormal vectors $\left\{\boldsymbol{\omega}_{n}\right\}_{n=0}^{N}$. In this letter we choose the vector $\boldsymbol{\omega}_{n}$ to be the $n$th column of the $T \times T$ discrete Fourier transform (DFT) matrix $\boldsymbol{F}$ with elements

$$
[\boldsymbol{F}]_{\ell, m}=e^{-2 \jmath \pi \ell m / T} .
$$

Since $\boldsymbol{F} \boldsymbol{F}^{\mathrm{H}}=\boldsymbol{I}$, the condition 8 holds 
In terms of the static part $\boldsymbol{\Omega}_{n}$, since in this letter we do not assume any prior knowledge of the user location, we set this part randomly. However, if it is assumed that an estimation of the user location and orientation is available, one can design $\Omega_{n}$ to obtain a higher signal-to-noise ratio (SNR) at the Rxs.

\section{B. ToA estimation at $B S m$}

In order to estimate $\tau_{n, m}$ at $\operatorname{Rx} m$ and for $n=0 \ldots N$, we make use of $(8)$ by computing

$$
\begin{aligned}
\boldsymbol{r}_{n, m} & =\frac{1}{T} \boldsymbol{Y}_{m} \boldsymbol{\omega}_{n}^{*} \\
& =\sqrt{E_{\mathrm{s}}} \beta_{n, m} \boldsymbol{d}\left(\tau_{n, m}\right)+\boldsymbol{w}_{n, m}
\end{aligned}
$$

where $\mathbb{E}\left\{\boldsymbol{w}_{0, m} \boldsymbol{w}_{0, m}^{H}\right\}=N_{0} / T \boldsymbol{I}$ and

$$
\beta_{n, m}= \begin{cases}\alpha_{0, m} & \text { if } n=0 \\ \gamma_{0, n} \gamma_{m, n} \boldsymbol{a}\left(\boldsymbol{\theta}_{m, n}\right)^{\mathrm{H}} \boldsymbol{\Omega}_{n} \boldsymbol{a}\left(\phi_{0, n}\right) & \text { otherwise }\end{cases}
$$

From this observation, we can easily determine $\tau_{n, m}$ using standard methods. In this letter we use fast Fourier transform (FFT) with a refinement step based on quasi-Newton method [13]. In what follows we explain this method in brief for completeness. We let $\boldsymbol{r}(\delta)=\boldsymbol{r} \circ \boldsymbol{d}(\delta)$ be a delayed version of $\boldsymbol{r}$. Also let $\boldsymbol{b}(\delta)$ be the $F$-point FFT of the vector $\boldsymbol{r}(\delta)$, where $F$ is a design parameter. Then we estimate $\tau_{n, m}$ as $\hat{\tau}_{n, m}=\tilde{k} / F \Delta f-\tilde{\delta}$, where $[\tilde{\delta}, \tilde{k}]=\arg \max _{k, \delta}\left|[\boldsymbol{b}(\delta)]_{k}\right|$. This 2D optimizations can be divided to two 1D ones [13].

\section{Estimating the position of user $n$}

We compute the TDoA measurements

$$
\begin{aligned}
\Delta_{n, m} & =c\left(\hat{\tau}_{n, m}-\hat{\tau}_{0, m}\right)+\left\|\boldsymbol{p}_{0}-\boldsymbol{p}_{m}\right\| \\
& =\left\|\boldsymbol{p}_{0}-\boldsymbol{x}_{n}\right\|+\left\|\boldsymbol{x}_{n}-\boldsymbol{p}_{m}\right\|+w_{n, m},
\end{aligned}
$$

which defines an ellipse in 2D and an ellipsoid 3D, with foci $\boldsymbol{p}_{0}$ and $\boldsymbol{p}_{m}$. For each UE $n$, we aggregate all the measurements in $\boldsymbol{\Delta}_{n}=\left[\Delta_{n, 1}, \ldots, \Delta_{n, M}\right]^{\top}$ across different Rxs and the corresponding noises in $\boldsymbol{w}_{n}$, where we model $\boldsymbol{w}_{n} \sim \mathcal{N}\left(\mathbf{0}, \boldsymbol{\Sigma}_{n}\right)$. Note that $\boldsymbol{\Sigma}_{n}$ is a diagonal matrix, since the noises at different Rxs are uncorrelated. The elements of $\boldsymbol{\Sigma}_{n}$ can be estimated using the CRB bounds for $\tau_{n, m}$ (see Section B. Hence, we can write

$$
\boldsymbol{\Delta}_{n}=\boldsymbol{h}\left(\boldsymbol{x}_{n}\right)+\boldsymbol{n}_{n}
$$

where

$$
[\boldsymbol{h}(\boldsymbol{x})]_{m}=\left\|\boldsymbol{p}_{0}-\boldsymbol{x}\right\|+\left\|\boldsymbol{x}-\boldsymbol{p}_{m}\right\| .
$$

We thus find the UE location estimate as

$$
\hat{\boldsymbol{x}}_{n}=\arg \min _{\boldsymbol{x}_{n}}\left(\boldsymbol{\Delta}_{n}-\boldsymbol{h}\left(\boldsymbol{x}_{n}\right)\right)^{\top} \boldsymbol{\Sigma}^{-1}\left(\boldsymbol{\Delta}_{n}-\boldsymbol{h}\left(\boldsymbol{x}_{n}\right)\right),
$$

which can be solved via gradient descent algorithm, starting from an initial guess. We next propose a method to find such an initial guess.

Without loss of generality, we set $\boldsymbol{p}_{0}=\mathbf{0}$. In the absence of noise and based on (14), we have that

$$
\left(\Delta_{n, m}-\left\|\boldsymbol{x}_{n}\right\|\right)^{2}=\left\|\boldsymbol{x}_{n}-\boldsymbol{p}_{m}\right\|^{2}
$$

TABLE I

PARAMETERS USED IN THE SIMULATION.

\begin{tabular}{lll}
\hline \hline Parameter & Symbol & Value \\
\hline Wavelength & $\lambda$ & $1 \mathrm{~cm}$ \\
RIS element distance & $d$ & $0.5 \mathrm{~cm}$ \\
Light speed & $c$ & $3 \times 10^{8} \mathrm{~m} / \mathrm{s}$ \\
Number of subcarriers & $N_{\mathrm{sc}}$ & 100 \\
Subcarrier bandwidth & $\Delta f$ & $120 \mathrm{kHz}$ \\
Number of transmissions & $T$ & 32 \\
Transmission Power & $N_{\mathrm{sc}} E_{\mathrm{s}} \Delta f$ & $20 \mathrm{dBm}$ \\
Noise PSD & $N_{0}$ & $-174 \mathrm{dBm} / \mathrm{Hz}$ \\
UE's Noise figure & $n_{f}$ & $8 \mathrm{~dB}$ \\
FFT dimensions & $F$ & 1024 \\
\hline \hline
\end{tabular}

which leads to

$$
\boldsymbol{p}_{m}^{\top} \boldsymbol{x}-\Delta_{n, m}\left\|\boldsymbol{x}_{n}\right\|=\frac{1}{2}\left(\left\|\boldsymbol{p}_{m}\right\|^{2}-\Delta_{n, m}^{2}\right) .
$$

We can rewrite (19) in the matrix form as

$$
\boldsymbol{P} \boldsymbol{x}_{n}=\boldsymbol{z}_{n}+\boldsymbol{\Delta}_{n}\left\|\boldsymbol{x}_{n}\right\|
$$

where $\boldsymbol{P}=\left[\boldsymbol{p}_{1}, \boldsymbol{p}_{2}, \ldots, \boldsymbol{p}_{M}\right]^{\top}, \boldsymbol{z}=0.5\left[\left\|\boldsymbol{p}_{1}\right\|^{2}-\right.$ $\left.\Delta_{n, 1}^{2}, \ldots,\left\|\boldsymbol{p}_{M}\right\|^{2}-\Delta_{n, M}^{2}\right]^{\top}$. Then the $n$th user position can be estimated as [14]

$$
\hat{\boldsymbol{x}}_{n}=\boldsymbol{a}_{n}+\boldsymbol{b}_{n}\left\|\hat{\boldsymbol{x}}_{n}\right\|
$$

where

$$
\begin{aligned}
\boldsymbol{a}_{n} & =\left(\boldsymbol{P}^{\top} \boldsymbol{P}\right)^{-1} \boldsymbol{P}^{\top} \boldsymbol{z}_{n} \\
\boldsymbol{b}_{n} & =\left(\boldsymbol{P}^{\top} \boldsymbol{P}\right)^{-1} \boldsymbol{P}^{\top} \boldsymbol{\Delta} \\
\left\|\hat{\boldsymbol{x}}_{n}\right\| & =\frac{-\boldsymbol{a}_{n}^{\top} \boldsymbol{b}_{n} \pm \sqrt{\left(\boldsymbol{a}_{n}^{\top} \boldsymbol{b}_{n}\right)^{2}-\left\|\boldsymbol{a}_{n}\right\|^{2}\left(\left\|\boldsymbol{b}_{n}\right\|^{2}-1\right)}}{\left\|\boldsymbol{b}_{n}\right\|^{2}-1} .
\end{aligned}
$$

If 24) yields two viable solutions, on can insert both solutions to the negative log-likelihood function, which is the objective function in (17). If the outcome for one of the solutions is much smaller than the other one, then it should be used as the initial guess for the $n$th user position. However, if both outcomes are small, this indicates that the $M$ ellipsoids in (14) intersects in two distinct points. In such a case some prior knowledge (e.g., the user is located below the Rxs) should be used to localize the user.

\section{Simulation Results}

In this section we evaluate the estimation error of the user position and compare it to the theoretical position error bound (PEB) (see Appendix). We let RIS to be a $64 \times 64$ uniform planar array (UPA). The clock biases $B_{m}$ are selected uniformly in the interval $[0,1 / \Delta f)$. Since there is no interference between the LOS path and the non line-of-sight (NLOS) paths from different users, the performance of the estimator for each user is independent of the number of users $N$ (as long as $T>N$ ) and therefore, we set $N=1$. For the LOS path, the channel gain $\alpha_{0, m}$ is calculated based on Friis' formula assuming unit directivity for Tx and Rxs. For the NLOS path the channel gain is calculated as [15. Eq. (21)-(22)]

$$
\gamma_{n, 0} \gamma_{n, m}=\frac{\lambda^{2}\left(\cos \left(\theta_{n, m}\right) \cos \left(\phi_{n, 0}\right)\right)^{0.285}}{16\left\|\boldsymbol{p}_{0}-\boldsymbol{x}_{n}\right\|\left\|\boldsymbol{p}_{m}-\boldsymbol{x}_{n}\right\|} .
$$




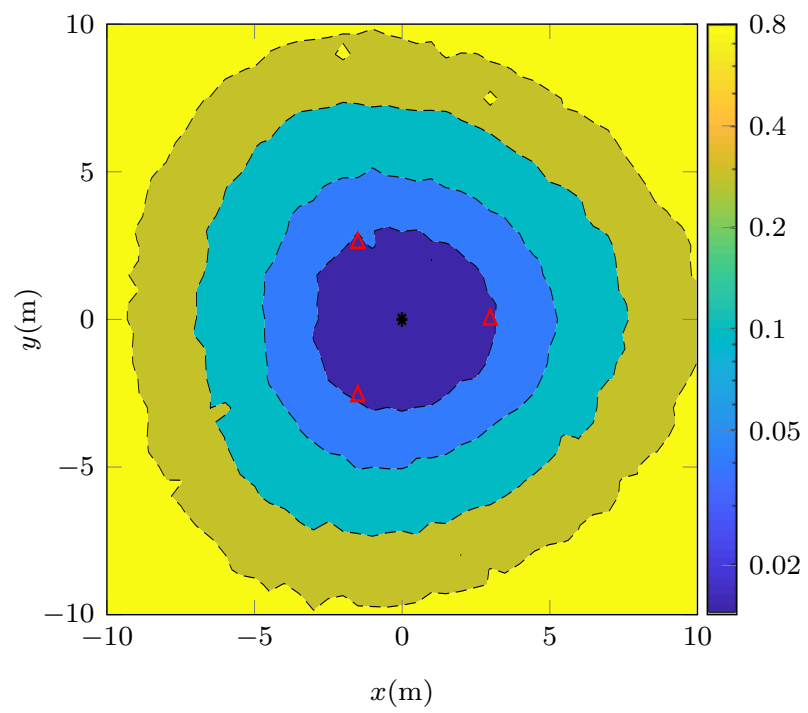

Fig. 2. PEB in meters for a system with $T x$ at the origin (marked by a black circle), three Rxs (marked by red triangles) uniformly located on a circle with radius 3 on the plane $z=1$, and a user located on $z=-3$.

All the rotational angles corresponding to the user orientation is set to zero $\left(\boldsymbol{R}_{n}=I_{3}, \forall n\right)$. The elements of $\boldsymbol{\Omega}_{n}$ are drawn randomly and independently from the unit circle. The presented results are obtained by averaging over 50000 random realization of RIS phase profiles (500) and noise (100 for each RIS phase profile). The rest of the system parameters are represented in Table $\mathrm{I}$.

Fig.2 illustrates the PEB for a system with three Rxs, located on the plane $z=1$, while the user is on $z=-3$. As can be seen, submeter localization accuracy can be attained in a large area around the Tx. It is also evident that PEB increases with the distance from the user to Tx almost symmetrically in all directions. In Fig. 3 the PEB is calculated as a function of the number of receivers $(M)$, where the receivers are located on a circle with radius $R \in\{2,4,6,8,10\}$ one meter above the Tx. and the user is at $\boldsymbol{x}_{1}=[0,0,-3]$. Naturally, PEB increases with $R$ due to a decrease in SNR and decreases with $M$. It can be seen that in the logarithmic scale, there is a linear dependence between $M$ and the position error (based on the numerical results in Fig. 3 the error is proportional to $\left.M^{-0.7}\right)$.

Fig. 4 compares the positioning error of the estimator presented in Section III-C with the CRB bounds, where the user is moved along the $x$ direction (we omit $1 \%$ of the RIS phase profiles as outliers). To assess the robustness of our algorithm to interference from the scatterers, we examine its performance in the presence of a number of scatterers. We place multiple scattered randomly one meter below the UEs and within $10 \mathrm{~m}$ reduce of the point $[0,0,-4]^{\top}$. The channel gain for the scattered signal is calculated based on the radar range equation by assuming radar cross section of $0.1 \mathrm{~m}^{2}$ (almost equal to the considered RIS surface area). The scatterers induce interference to the received signal at every Rxs. Such interference will deteriorate our estimation accuracy of the LOS delay $\hat{\tau}_{0, m}$, however, it does not effect that of the NLOS

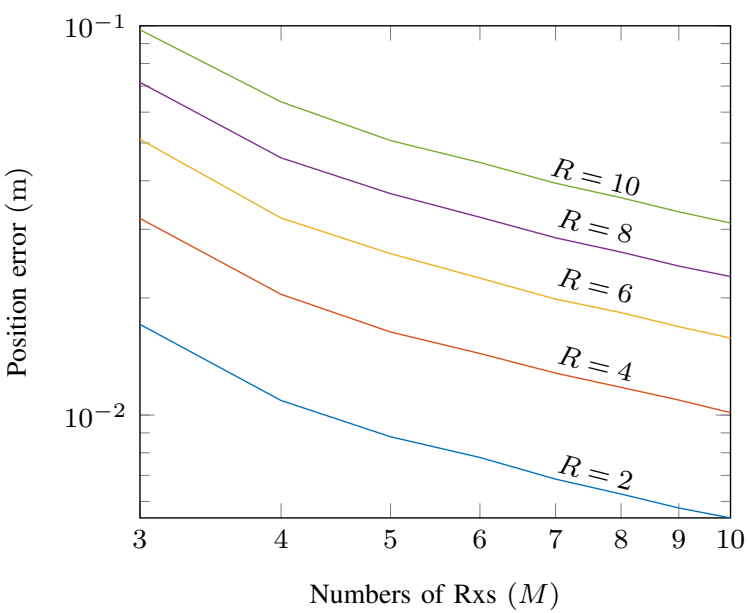

Fig. 3. PEB for a system with Tx at the origin, $M$ Rxs located uniformly in a circle of radius $R$ on the $z=1$ plane, and a user located at $[0,0,-3]$.

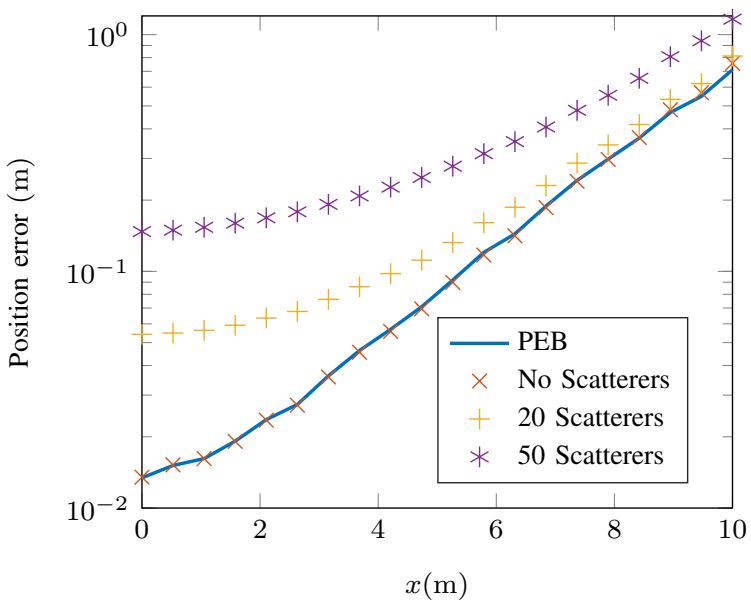

Fig. 4. PEB (solid line) for a system with Tx at the origin, three Rxs (located similarly as in Fig. 2), and a user located at $[x, 0,-3]$. The estimation error is also shown by markers in the presence of 0,20 , and 50 scatterers.

delay $\hat{\tau}_{n, m}(n>0)$. This is because the interference from scatterers cancels out upon calculating $\boldsymbol{r}_{n, m}=(1 / T) \boldsymbol{Y}_{m} \boldsymbol{\omega}_{n}^{*}$ since we have $\mathbf{1}_{T}^{\top} \boldsymbol{\omega}_{n}^{*}=0, \forall n>0$. In the absence of scatterers, it can be seen that the estimator is able to reach the theoretical bound. We use the prior knowledge that the UE is below the Rxs to resolve the sign ambiguity in (24). Furthermore, it can be seen that the estimator can function properly even in the presence of a large number of scatterers and obtain submeter localization accuracy. When the user is close to the transmitter and SNR is high, the interference becomes the limiting factor and the effects of scatterers are more pronounced. On the other hand, the effect of interference becomes minimal in the lowSNR regime where the noise limits the performance the the estimator.

\section{CONCLUSION}

We considered a multi-user RIS-enabled localization problem, where the users' position in $3 \mathrm{D}$ was estimated by calculating the ToA of the LOS and NLOS paths at multiple receivers. The considered scenario can be categorized as a 
passive localization problem since the users do not transmit or receive signals and their positions are obtained based on the reflected signal from them. Nonetheless, it should be noted that RISs are not completely passive as they require some source of energy to reconfigure. We showed that by dividing the RIS phase profile to dynamic and static parts and selecting the dynamic one based on orthonormal sequences, the interpath interference can be avoided. In future work we aim to optimize the static part of the RIS phase profile to improve the SNR of the NLOS path and achieve better localization accuracy.

\section{APPENDIX}

\section{CRB CALCULATIONS}

\section{A. Calculating CRB for $\tau_{n, m}$}

The CRB for $\tau_{n, m}$ is a lower bound on the estimation error of the ToA, i.e.,

$$
\sqrt{\mathrm{E}\left[\left|\tau_{n, m}-\hat{\tau}_{n, m}\right|^{2}\right]} \geq \sqrt{\left[\boldsymbol{J}_{n, m}^{-1}\right]_{1,1}}
$$

where $\boldsymbol{J}_{n, m}$ is the Fisher information matrix (FIM) for the channel parameters

$$
\boldsymbol{\zeta}_{n, m}=\left[\tau_{n, m}, \Re\left(\beta_{n, m}\right), \Im\left(\beta_{n, m}\right)\right]
$$

The FIM can be calculated as

$$
\boldsymbol{J}_{n, m}=\frac{2 T}{N_{0}} \sum_{k} \Re\left(\frac{\partial\left[\boldsymbol{r}_{n, m}\right]_{k}}{\boldsymbol{\zeta}_{n, m}}\left(\frac{\partial\left[\boldsymbol{r}_{n, m}\right]_{k}}{\boldsymbol{\zeta}_{n, m}}\right)^{\mathrm{H}}\right)
$$

Then, we can calculate the elements of $\boldsymbol{J}_{n, m}$ as

$$
\begin{aligned}
& {\left[\boldsymbol{J}_{n, m}\right]_{1,1}=\frac{2 K T E_{\mathrm{s}}}{N_{0}}\left|2 \pi \Delta f \beta_{n, m}\right|^{2} \frac{(K-1)(2 K-1)}{6}} \\
& {\left[\boldsymbol{J}_{n, m}\right]_{1,2}=-\frac{2 K T E_{\mathrm{s}}}{N_{0}} 2 \pi \Delta f \Im\left(\beta_{n, m}\right) \frac{K-1}{2}} \\
& {\left[\boldsymbol{J}_{n, m}\right]_{1,3}=\frac{2 K T E_{\mathrm{s}}}{N_{0}} 2 \pi \Delta f \Re\left(\beta_{n, m}\right) \frac{K-1}{2}} \\
& {\left[\boldsymbol{J}_{n, m}\right]_{2,3}=0} \\
& {\left[\boldsymbol{J}_{n, m}\right]_{2,2}=\frac{2 K T E_{\mathrm{s}}}{N_{0}}} \\
& {\left[\boldsymbol{J}_{n, m}\right]_{3,3}=\frac{2 K T E_{\mathrm{s}}}{N_{0}}}
\end{aligned}
$$

Base on (28)-(34), we have

$$
\left[\boldsymbol{J}_{n, m}^{-1}\right]_{1,1}=\frac{N_{0}}{2 K T E_{\mathrm{s}}}\left|2 \pi \Delta f \beta_{n, m}\right|^{-2} \frac{12}{K^{2}-1}
$$

\section{B. Calculating $\boldsymbol{\Sigma}_{n}$}

Based on (13), the covariance matrix $\Sigma_{n}$ can be calculated using (35) as

$$
\left[\boldsymbol{\Sigma}_{n}\right]_{m, m}=c^{2}\left(\left[\boldsymbol{J}_{n, m}^{-1}\right]_{1,1}+\left[\boldsymbol{J}_{0, m}^{-1}\right]_{1,1}\right)
$$

where in order to calculate 35 we estimate $\left|\beta_{n, m}\right|$ based on (11) as

$$
\left|\beta_{n, m}\right|=\left|\frac{\boldsymbol{d}\left(\hat{\tau}_{n, m}\right)^{\top} \boldsymbol{r}_{n, m}}{\sqrt{E_{\mathrm{s}}} \boldsymbol{d}\left(\hat{\tau}_{n, m}\right)^{\top} \boldsymbol{d}\left(\hat{\tau}_{n, m}\right)}\right|
$$

\section{Calculating PEB}

Based on [16. Eq.(3.31)] and (14) we have

$$
\mathrm{PEB}_{n}=\sqrt{\operatorname{tr}\left(\boldsymbol{J}_{n}^{-1}\right)}
$$

where

$$
\begin{aligned}
\boldsymbol{J}_{n}= & \frac{\partial \boldsymbol{\mu}}{\partial \boldsymbol{x}_{n}} \Sigma^{-1}\left(\frac{\partial \boldsymbol{\mu}}{\partial \boldsymbol{x}_{n}}\right)^{\top}, \\
\frac{\partial \boldsymbol{\mu}}{\partial \boldsymbol{x}_{n}}= & {\left[\frac{\boldsymbol{x}_{n}-\boldsymbol{p}_{0}}{\left\|\boldsymbol{x}_{n}-\boldsymbol{p}_{0}\right\|}+\frac{\boldsymbol{x}_{n}-\boldsymbol{p}_{1}}{\left\|\boldsymbol{x}_{n}-\boldsymbol{p}_{1}\right\|},\right.} \\
& \left.\ldots, \frac{\boldsymbol{x}_{n}-\boldsymbol{p}_{0}}{\left\|\boldsymbol{x}_{n}-\boldsymbol{p}_{0}\right\|}+\frac{\boldsymbol{x}_{n}-\boldsymbol{p}_{M}}{\left\|\boldsymbol{x}_{n}-\boldsymbol{p}_{M}\right\|}\right] .
\end{aligned}
$$

\section{REFERENCES}

[1] D. Dardari, "Communicating with large intelligent surfaces: Fundamental limits and models," IEEE J. Select. Areas Commun., vol. 38, no. 11, pp. 2526-2537, Nov. 2020.

[2] A. Bourdoux, A. N. Barreto, B. van Liempd, C. de Lima, D. Dardari, D. Belot, E.-S. Lohan, G. Seco-Granados, H. Sarieddeen, H. Wymeersch et al., "6G white paper on localization and sensing," arXiv preprint arXiv:2006.01779, Jun. 2020.

[3] H. Wymeersch, J. He, B. Denis, A. Clemente, and M. Juntti, "Radio localization and mapping with reconfigurable intelligent surfaces: Challenges, opportunities, and research directions," IEEE Vehicular Technology Magazine, vol. 15, no. 4, pp. 52-61, Dec. 2020.

[4] L. M. Ni, D. Zhang, and M. R. Souryal, "RFID-based localization and tracking technologies," IEEE Wireless Commun., vol. 18, no. 2, pp. 4551, Apr. 2011.

[5] H. Qin, Y. Peng, and W. Zhang, "Vehicles on RFID: Error-cognitive vehicle localization in GPS-less environments," IEEE Trans. Vehicular Tech., vol. 66, no. 11, pp. 9943-9957, Nov. 2017.

[6] J. Hong and T. Ohtsuki, "Signal eigenvector-based device-free passive localization using array sensor," IEEE Trans. Vehicular Tech., vol. 64, no. 4, pp. 1354-1363, Apr. 2015.

[7] W. Ruan, L. Yao, Q. Z. Sheng, N. J. Falkner, and X. Li, "Tagtrack: Device-free localization and tracking using passive RFID tags," in Proceedings of the 11th Int. Conf. on Mobile and Ubiquitous System, London, UK, Dec. 2014, pp. 80-89.

[8] J. Shen, A. F. Molisch, and J. Salmi, "Accurate passive location estimation using TOA measurements," IEEE Trans. Wireless Commun., vol. 11, no. 6, pp. 2182-2192, Jun. 2012.

[9] Y. Wang, S. Ma, and C. P. Chen, "TOA-based passive localization in quasi-synchronous networks," IEEE Commun. Lett., vol. 18, no. 4, pp. 592-595, Feb. 2014.

[10] W. Yuan, N. Wu, B. Etzlinger, Y. Li, C. Yan, and L. Hanzo, "Expectation-maximization-based passive localization relying on asynchronous receivers: Centralized versus distributed implementations," IEEE Trans. Commun., vol. 67, no. 1, pp. 668-681, Jan. 2019.

[11] N. Decarli, F. Guidi, and D. Dardari, "A novel joint RFID and radar sensor network for passive localization: Design and performance bounds," IEEE J. Select. Areas Commun., vol. 8, no. 1, pp. 80-95, Feb. 2014.

[12] X. Zhang, H. Li, J. Liu, and B. Himed, "Joint delay and Doppler estimation for passive sensing with direct-path interference," IEEE Transactions on Signal Processing, vol. 64, no. 3, pp. 630-640, 2016.

[13] K. Keykhosravi, M. F. Keskin, G. Seco-Granados, and H. Wymeersch, "SISO RIS-enabled joint 3D downlink localization and synchronization," arXiv preprint arXiv:2011.02391, 2020.

[14] M. Malanowski, "An algorithm for 3D target localization from passive radar measurements," in Photon. Appl. in Astron., Commun., Industry, and High-Energy Phys. Exp., Wilga, Poland, May 2009.

[15] S. W. Ellingson, "Path loss in reconfigurable intelligent surface-enabled channels," arXiv preprint arXiv:1912.06759, 2019.

[16] S. M. Kay, Fundamentals of statistical signal processing: Estimation Theory. Prentice Hall PTR, 1993. 